\title{
Pourquoi dépasser l'image mécaniste du Charlot des Temps modernes? K. Burke, le travail et le pouvoir des images
}

Why shoud the mechanistic image of Modern Times' Charlot be overtaken? K.

Burke, work and the power of pictures

\section{Alexandra Bidet}

\section{OpenEdition}

\section{Journals}

Édition électronique

URL : http://journals.openedition.org/itti/1366

DOI : 10.4000/itti. 1366

Éditeur

Université de Poitiers

Référence électronique

Alexandra Bidet, « Pourquoi dépasser l'image mécaniste du Charlot des Temps modernes ? K. Burke, le travail et le pouvoir des images », Images du travail, travail des images [En ligne], 1 | 2016, mis en ligne le 01 février 2016, consulté le 14 avril 2021. URL : http://journals.openedition.org/itti/1366 ; DOI : https:// doi.org/10.4000/itti.1366

Ce document a été généré automatiquement le 14 avril 2021.

Images du travail, travail des images 


\section{Pourquoi dépasser l'image mécaniste du Charlot des Temps modernes? K. Burke, le travail et le pouvoir des images}

Why shoud the mechanistic image of Modern Times' Charlot be overtaken? K. Burke, work and the power of pictures

\section{Alexandra Bidet}

Une image s'impose pour illustrer le travail: le Charlot des Temps modernes de Charlie Chaplin ${ }^{1}$. Son omniprésence, sur les couvertures d'ouvrages et les affiches de rencontres scientifiques ou grand public sur le travail, contraste avec la tendance séculaire à la tertiarisation et l'informatisation des activités, qui voit les salariés moins impliqués dans des actions mécaniques, que dans le maniement d'outils informatiques et de relations humaines. Dans les interactions de service, les relations clients, les coopérations en mode projet ou réseau, les processus automatisés (nucléaire, aviation, pétrochimie, télécommunications, agro-alimentaire...), le travail humain est davantage mobilisé pour sa plasticité, sa capacité d'adaptation, que pour sa force motrice. Le passage d'un paradigme de la charge à soulever à un paradigme de l'aléa à gérer s'observe dès les années 1950 (Naville, 1961), Or, nous sommes - nous chercheurs, observateurs, représentants, acteurs du travail - tellement habitués à associer le travail au Charlot des Temps modernes que prenons encore mal la mesure de ces activités en plein essor: vigilance, exploration, tact interactionnel, coordination, gestion d'engagements, etc. ${ }^{2}$. Elles appellent à réinventer nos façons de figurer le travail. 
Illustration 1 : Couverture du « Que Sais-je ? ", Le Travail

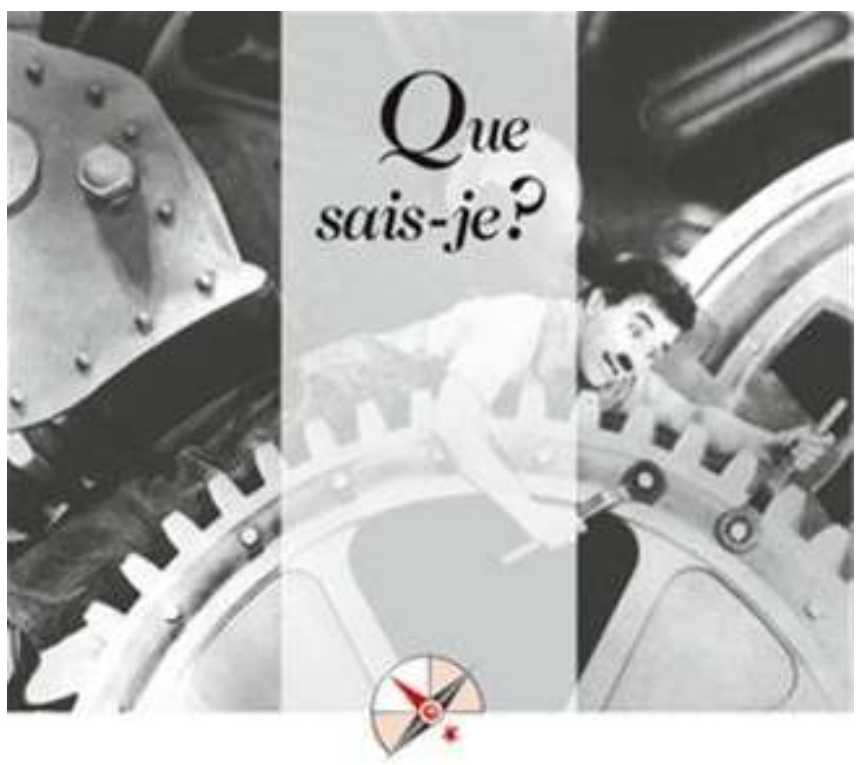

\section{LE TRAVAIL}

Méda Dominique, Le travail, 4e éd., Paris, Presses Universitaires de France « Que sais-je? », 2010

2 L'image de Charlot ne réduit pas seulement le travail à l'un de ses usages. Elle le rabat aussi sur une pure contrainte. En ignorant ainsi l'ambivalence observée jusque dans les contextes les plus contraints, elle conforte les conceptualisations les plus communes, qui identifient le travail à l'exécution de consignes, sans créativité ni mobilisation personnelle (Bidet, 2011a). A l'heure où la créativité du travail humain et ses capacités d'adaptation sont plus sollicitées que jamais, ses activités peinent donc à être figurées, et a fortiori reconnues. Les représentations sociales dominantes du travail, issues du XIXe siècle industriel, tendent plutôt à dissoudre les activités de travail contemporaines, aux yeux même des travailleurs, qui, faute d'une dépense de force ou d'un produit matériel tangible, peuvent se demander s'ils travaillent vraiment.

Cet article ne propose pas une nouvelle iconographie clefs en main du travail. Il faudra un intense commerce entre artistes, observateurs, représentants et acteurs du travail, pour relever ce défi. Il cherche plutôt à éclairer la prégnance de cette image mécaniste et l'intérêt de s'en défaire, en poursuivant quelques pistes. En particulier, celle ouverte par le critique littéraire et philosophe Kenneth Burke (1897-1993), qui appelle à substituer à la métaphore mécaniste une métaphore poétique. Auteur d'une œuvre largement consacrée à nos façons de fabriquer des symboles, d'en user et mésuser, $\mathrm{K}$. Burke souligne le caractère vital de ces « équipements de nos vies », qui répondent à la nécessité de s'orienter. Mais aussi l'intérêt d'en mener une critique, de tester collectivement leur pertinence, afin d'éviter qu'ils nous abusent à mesure que croît, avec la division du travail et l'urbanisation, la complexité de nos sociétés. 
Illustration 2 et 3 : Affiche des cycles de débats de l'EHESS (au dessus) et affiche du colloque "L'activité en débat » (en dessous).

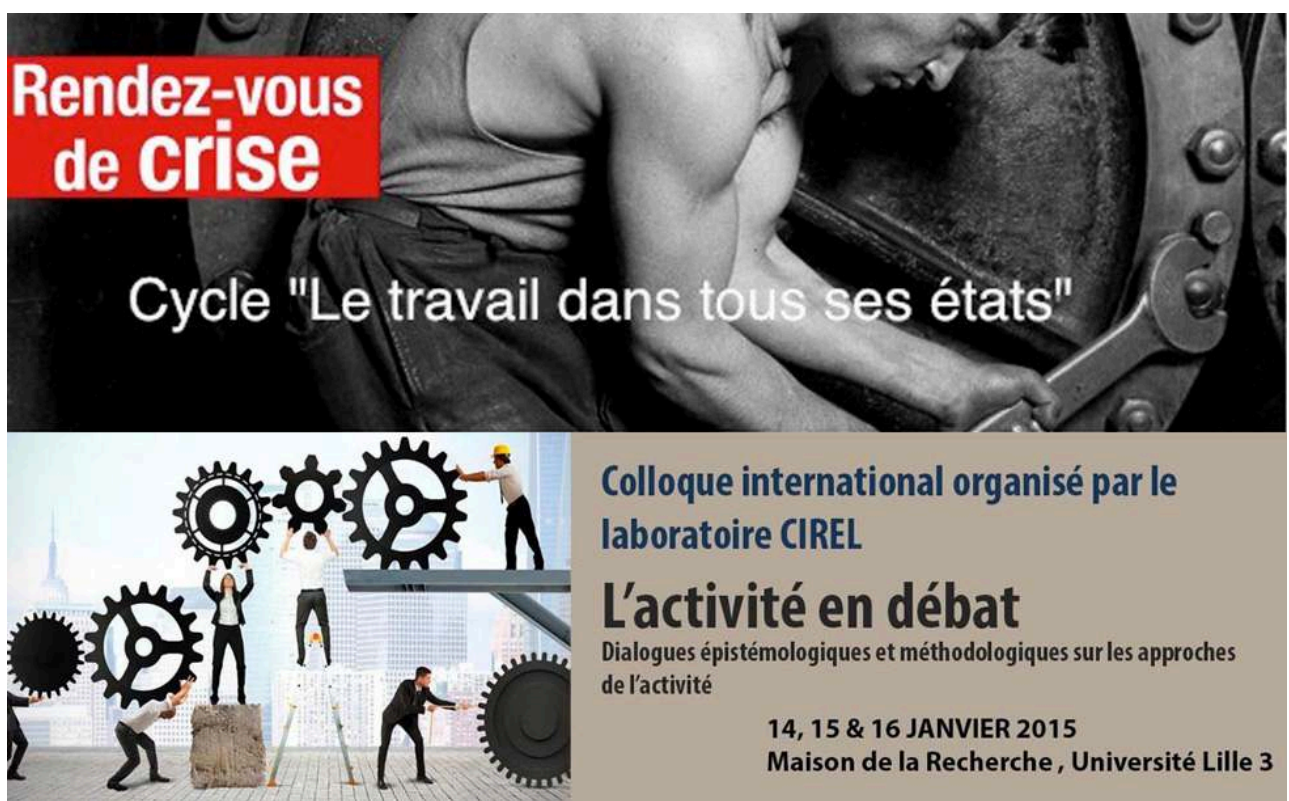

Illustration 4 : Couverture de l'ouvrage, Quel travail voulons-nous ? La grande enquête. (à gauche)

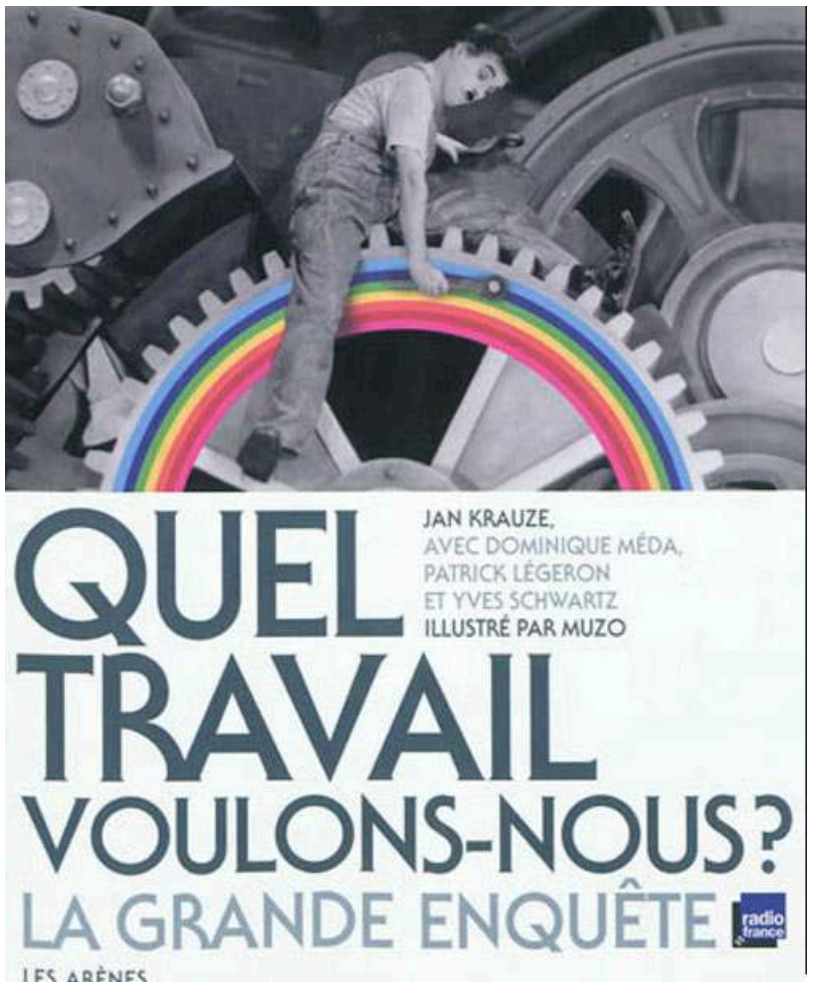

LES ARENEES

Jan Krauze, Dominique Méda, Patrick Légeron, Yves Schwartz, Quel travail voulons-nous ? La grande enquête, Paris, Les Arènes, 2012 


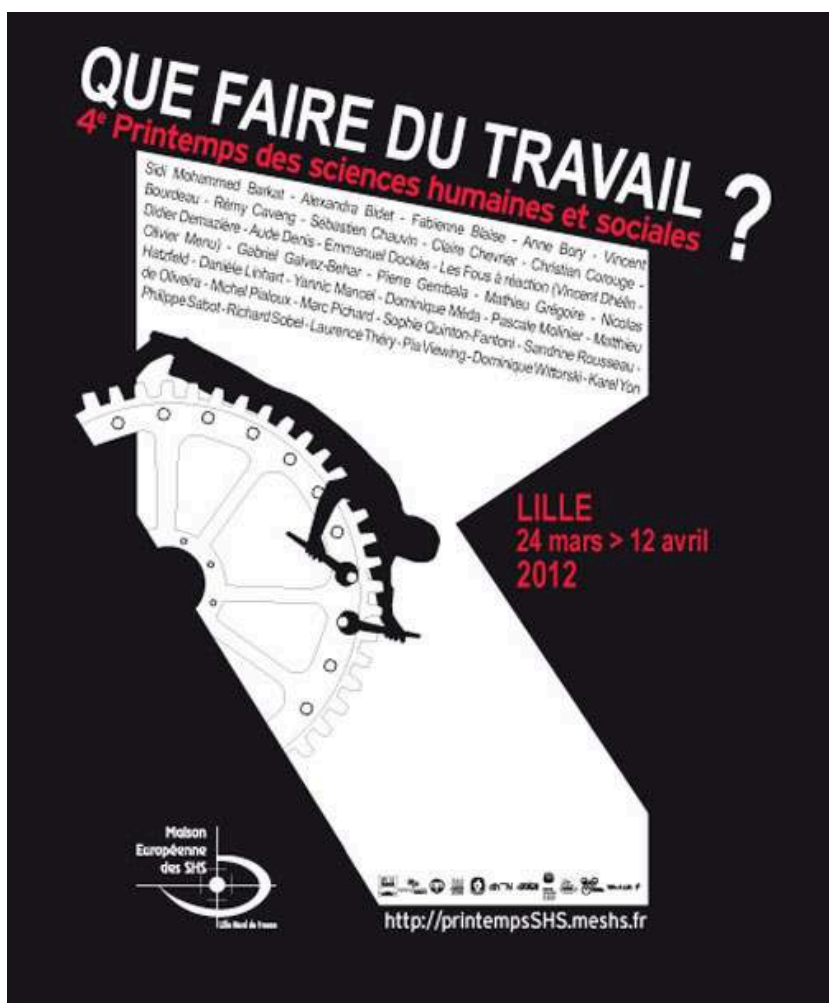

Maison européenne des sciences de l'homme et de la société Lille Nord de France

\section{Le travail comme force motrice : puissance d'une image anachronique}

K. Burke peut nous aider à comprendre la puissance d'attraction de Charlot, la surprenante persistance d'une image du travail pourtant périmée depuis l'invention de la machine à vapeur, du moins comme représentation générale des usages du travail humain (Vatin, 1999).

Rappelons que la représentation mécaniste ou physicaliste du travail trouve son origine dans un chassé-croisé entre l'observation du travail humain développée au XVIe et XVIIe siècle et l'élaboration d'une science des machines à la fin du XVIIIe siècle (Vatin, 1993). Si le travail humain en est venu à être considéré comme une quantité d'effort, une dépense de force énergétique, c'est que l'élaboration du concept de travail (mécanique) par les physiciens, comme le produit d'une force par une distance, a fait glisser le regard de l'homme vers la machine, du travail humain vers le travail mécanique, avant qu'il ne revienne vers l'homme à travers les pratiques d'organisation $\mathrm{du}$ travail en plein essor dès la fin du XIXe siècle. Le travail humain est alors appréhendé comme un travail mécanique. L'usage du travail humain comme simple force, anachronique depuis l'invention de la machine à vapeur, est depuis en constante régression. Des historiens comme J.-P. Daviet rappellent qu'au plus fort de la rationalisation industrielle, le travail à la chaîne taylorien, au sens strict, ne représente jamais plus de $5 \%$ des ouvriers en général (Vatin, 1999, p. 104). Depuis, l'expression d'« organisations tayloriennes » a été dissociée d'une référence directe à la pensée de $\mathrm{F}$. 
W. Taylor et à sa méthode d'analyse du travail, à un point tel qu'on peut douter de son sens (Vatin p. 87-106). Encore assouplie par la thématisation d'un "taylorisme flexible » ou "assisté par ordinateur », elle pourrait concerner de nos jours $14 \%$ des salariés (Lorenz, Valeyre, 2005, p. 96).

La fortune de la métaphore mécaniste dépasse toutefois les méthodes tayloriennes d'organisation du travail. Bien avant l'ouvrage de B. Trentin (2012), qui pointe la profonde séduction exercée par le fordisme et cet « imaginaire de l'homme automate » sur les pensées de gauche, $K$. Burke rappelait combien l'âge des Lumières a valorisé la machine (" ethicized machinery "), et incité à confondre mécanisation et progrès : " nous avons eu tendance à voir dans la machine un bien absolu, en identifiant fréquemment mécanisation et progrès (...) La machine avait un prestige tel que l'on s'efforçait d'importer cette métaphore dans d'autres champs d'investigation, en lui prêtant un pouvoir interprétatif absolu ou universel, comme lorsqu'on l'emploie pour 'expliquer' des types de comportements biologiques complètement différents des comportements mécaniques »(Burke, 1983, p. 207)3.

Illustration 6 : Couverture de l'ouvrage Le Travail invisible. Enquête sur une disparition.

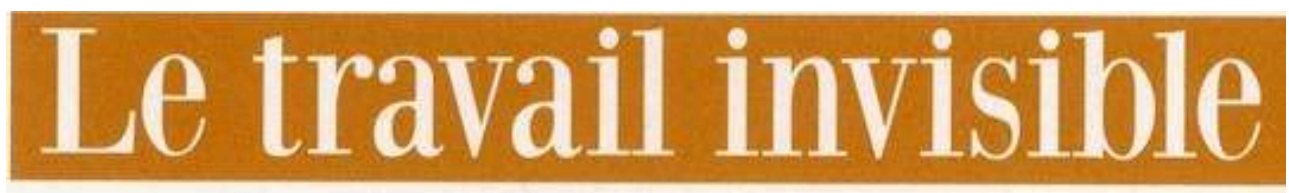

\section{Enquête sur une disparition}

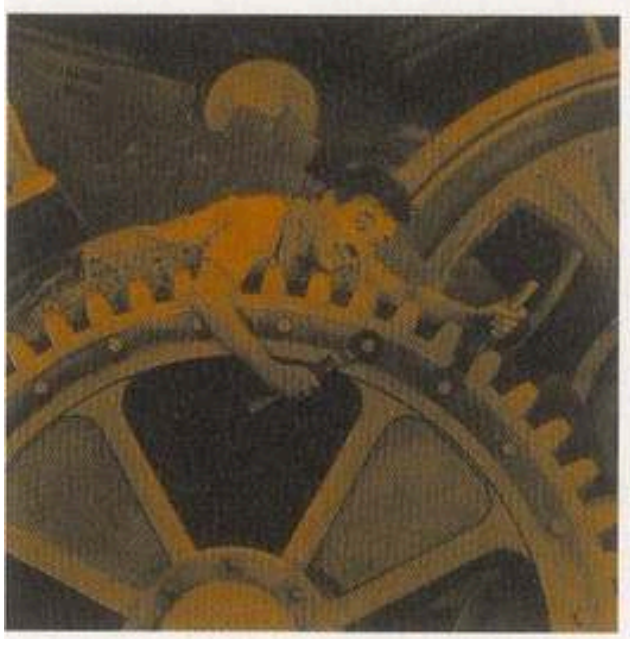

Pierre-Yves Gomez, Le travail invisible. Enquête sur une disparition, François Bourin Éditeur, 2013

La métaphore mécaniste est ainsi au principe de ce que $\mathrm{K}$. Burke nomme, à la suite de Th. Veblen (Wais, 2005), une "trained incapacity», une distorsion ou un aveuglement (incapacity) inhérents à l'acquisition d'une habileté (trained). La métaphore mécaniste, en apprenant à voir le travail à travers la machine, rend veugle à d'autres dimensions de l'agir humain, en particulier, à sa dimension poétique, par laquelle il produit sans cesse de nouvelles significations (Bidet, 2007b). Pour corriger cette tendance, K. Burke suggère de faire place à une métaphore poétique. 
8 Avant d'examiner cette proposition, illustrons la "trained incapacity ", qui conduit en effet la littérature académique, dans le domaine qui nous intéresse, à prêter peu d'attention aux transformations concrètes du contenu du travail. Alors que le travail humain est désormais peu sollicité pour sa capacité énergétique, les discours et les recherches sur le travail restent dominés par un lexique de l'intensification, de l'intensité, de la charge, de la productivité, du temps de travail, qui n'a de sens que dans un paradigme mécaniste. Le travail y est ramené à une quantité (mesurée en temps ou en énergie) et saisi négativement : désutilité, contrainte, effort pénible, dépense, coût à réduire (Bidet, Vatin, 2009). La force de cette image empêche encore de reconnaître pleinement le statut de travail aux activités, notamment, de lecture (Moatty, Rouard, Teiger, 2007), de vigilance, de communication, de tact interactionnel, qui engagent autrement le corps, et moins spontanément en termes d'effort pénible. La force de cette image de Charlot comme symbole d'une critique du travail pèse aussi sur les possibilités de renouvellement de la critique, encore très attachée à une image qui l'a longtemps conduite à privilégier l'emploi sur le travail. Sur les deux affiches reproduites ci-dessous, le projet de « réinventer le travail » ou d' « exister au travail » ne se sépare pas d'un fond d'écrous.

Illustration 7 et 8 : Affiche de Réinventer le travail des semaines sociales de France (à gauche) et numéro $n^{\circ}$ 26, Exister au travail de novembre/décembre 2013 de la revue Vie chrétienne (à droite)

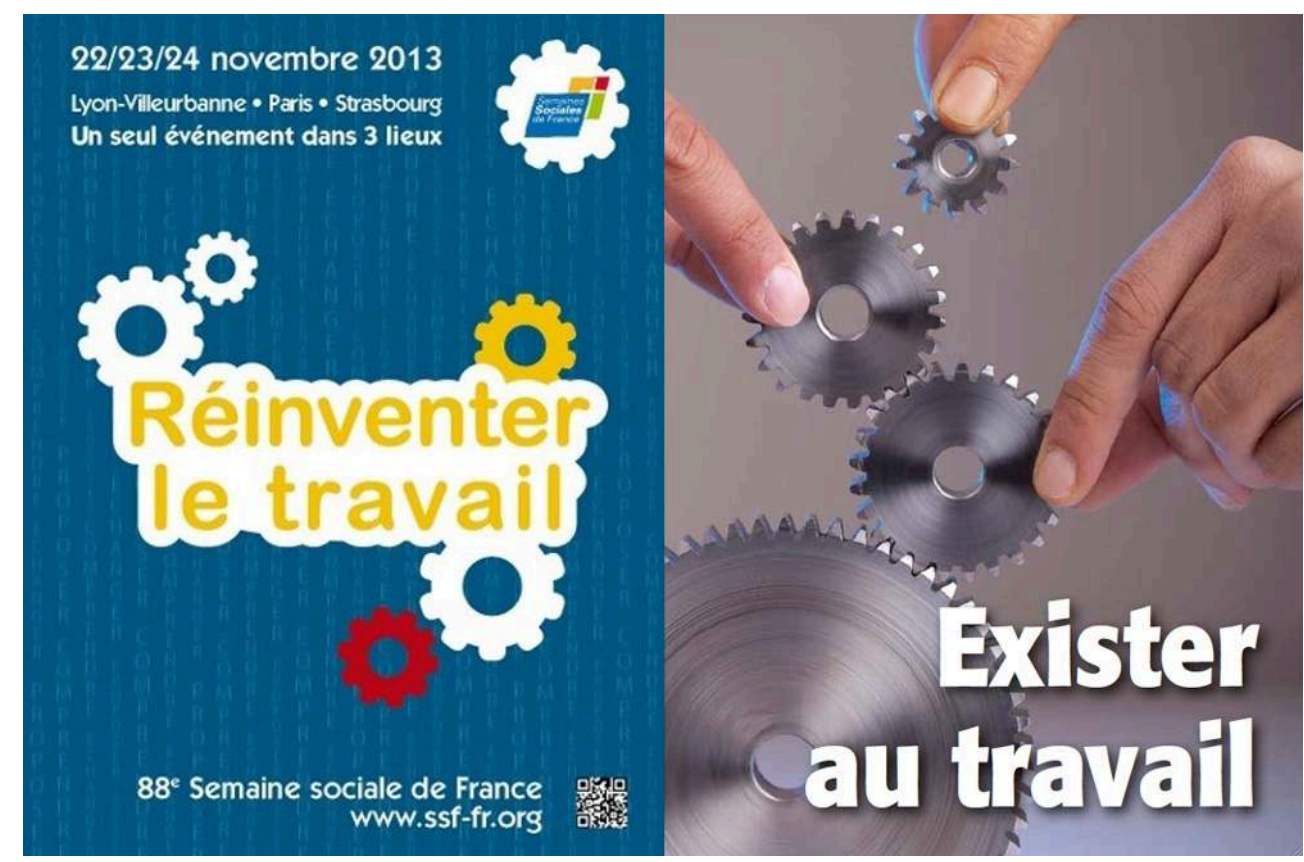

Explorer une autre métaphore, poétique, pourrait donc favoriser un renouvellement des modalités de critique du travail, mais aussi une plus large introduction du travail dans l'espace public, où il ne s'avance encore le plus souvent que sur un mode défensif et doloriste (Clot, 2010 ; Bidet, Vatin, 2013).

\section{Une métaphore poétique : saisir ce qui compte depuis le travail}

«Il semblerait que la rationalisation scientifique ne puisse être corrigée que par une logique artistique - non pas l'art d'un performer, ou d'un spécialiste, que 
quelques uns produisent et beaucoup observent, mais un art de vivre " " 1983, p. 66, notre traduction).

10 Pour éclairer ce que K. Burke entend par métaphore poétique, partons de sa réflexion sur la notion d'intérêt. Critique de l'utilitarisme et du calcul des plaisirs et des peines à la Bentham, K. Burke emprunte à J. Dewey la « subtile distinction entre ce qui est dans l'intérêt d'une personne et ce à quoi elle s'intéresse » (Burke, 1983, p. 45) : "le seul fait que quelque chose soit dans l'intérêt d'une personne ne garantit pas qu'elle s'y intéressera » (p. 38). Pour K. Burke, comme pour J. Dewey, l'activité de travail crée tout particulièrement ce second type d'intérêts. Dans la mesure où elle nous occupe durablement, elle suscite ce que J. Dewey nomme une "occupational psychosis " [déformation professionnelle]: "une orientation marquée de l'esprit», " une certaine manière de penser, allant avec une certaine façon de vivre ", qui peut être « véhiculée par le mot d'argot le plus ordinaire » (p. 38, 40). Travailler implique de délimiter « des ensembles d'objets et de relations qui importent, lesquels vont fournir à notre attention son contenu et sa matière, et définir les qualités qui nous paraîtront intéressantes ». Si un travail n'est initialement qu'un moyen, il devient ainsi une fin à mesure que nous créons autour de lui une façon de penser et de vivre à laquelle nous tenons: un "réseau moral» $[$ a moral network]. K. Burke appelle "peripheral charging " [investissement périphérique] ce processus qui voit chacun développer des « accents, des normes, des désirs, des manières d'observer, d'exprimer, de refouler, qui vont l'équiper pour sa tâche». Quand "travail et morale sont indémélables », alors «notre occupation professionnelle a atteint le stade de la préoccupation ». Elle est un «bien-en-soi »: «car ce qui nous préoccupe est quelque chose que nous valorisons [value] : une femme, une entreprise, un livre. Nous disons que nous valorisons [ethicize] une chose quand nous la traitons comme un bien en soi » (p. 238). Pour explorer et documenter ce lien entre travail et valorisations, et la tendance de ces dernières à s'auto-perpétuer, j’ai introduit la notion de « vrai boulot » (Bidet, 2011a).

11 "Occupational psychosis ", " ethicizing of work ", " vrai boulot ", cette genèse normative s'ancre dans une nécessité anthropologique : s'orienter, être un participant. L'espèce humaine ne peut en effet habiter le monde qu'en créant son propre environnement (Bidet, 2007 a, b), c'est-à-dire en y extériorisant des intérêts, "ce qui nous conduit à certains moments à déceler ce qui, dans les évènements, reflète immédiatement nos intérêts, et à d'autres moments, à nous concentrer sur un ensemble de moyens pour que les évènements reflètent nos intérêts » (p. 254)5 - découverts en route. Développer des manières de faire, c'est ainsi, se découvrir des intérêts en transformant le monde : « Les hommes 'socialisent' leurs modes spécifiques d'intérêts à travers la manipulation de matériaux concrets, laquelle rend l'intérieur et l'extérieur, pour un temps indéfini, indissociables » (p. 215).

12 Cette fusion entre extérieur et intérieur, très pragmatiste d'inspiration (Bidet, Boutet, Chave, 2013 ; Bidet, Chave, 2015), nous amène à la métaphore poétique : « La vie est un poème au sens où, au cours de notre vie, nous tissons peu à peu une structure de liens qui fait écho à nos intérêts ", écrit K. Burke (p. 254). Dans la mesure où le travail «à la fois, reflète nos intérêts et les forme ", il contribue à ce tissage d'une unité qui vaut par elle-même: "le poème est une soudaine fusion, un amalgame de nombreux éléments auparavant séparés - et la force même de cette fusion conduit à rechercher d'autres expériences de la même qualité » (p. 158). K. Burke prend l'exemple de la médecine : « on peut avoir été amené à embrasser la médecine par intérêt pour la médecine - mais quand on se fait à sa discipline, alors elle devient créative et nous offre de nouvelles 
incitations à voir toute la vie 'de point de vue du médecin'« (p. 240). De même, le boxeur en vient à percevoir le monde à travers ses poings : « le jeune boxeur valorisera tellement ses poings qu'il aura tendance à simplifier des relations humaines nombreuses et variées en faisant comme s'il pouvait y répondre par un coup porté au menton, ou par cette menace» (p. 242-243). Produire une vision du monde depuis son travail est une propension générale : « tous les hommes sont des poètes, y compris dans les sortes d'action que nous considérons généralement comme distinctes de la poésie » (p. 259).

13 La nécessité de participer, de s'orienter efficacement dans son activité, conduit chacun à « former une orientation », " un faisceau de jugements sur les façons dont les choses ont été, sont et peuvent être " (p. 14), ou "une nouvelle façon de mettre les choses ensemble, telle chaque ligne d'un drame» (p. 254). Chaque "orientation», dans la mesure où elle incite à laisser de côté ce qui lui échappe, est alors source de «trained incapacity»: " une façon de voir est aussi une façon de ne pas voir» (p. 49). Cette multiplicité de " visions professionnelles », partiales et partielles, ancrées à la fois dans des activités et dans des façons de les mener, fait le caractère fragmenté des mondes du travail. K. Burke craignait cette altérité grandissante, où chacun devient toujours plus un «mystère pour les autres", mais il redoutait aussi la myopie inhérente au confinement dans une vision professionnelle, qui n'est pas sans rappeler l'« idiotisme de métier $»^{6}$. K. Burke envisage ainsi que nous soyons " tellement hypnotisés et pris par la texture de notre poème, objectivé par des relations extérieures établies ", que même les autres ne pourraient que nous aider à y persévérer (p. 78). Contrairement à Th. Veblen, il ne s'en tient toutefois pas à la critique cet atavisme (Wais, 2005). Le jeu croisé des «trained incapacities» (qu'il ne circonscrit pas à l'orientation financière des hommes d'affaires, mais généralise à toute activité professionnelle) revêt chez lui une importance au moins égale, et indique une autre voie, celle de possibles "correctifs». Car notre "orientation", comme "trained incapacity» se heurte nécessairement à la résistance de ce qui lui échappe, aux choses et aux autres ; les « découvertes » que nous sommes ainsi amenés à faire ne sont alors « rien d'autre que des révisions appelées par la nature du monde lui-même » (p. 257). Intégrer sa résistance donne l'opportunité de transformer de vieux schèmes d'orientation et de "pseudo-énoncés " en «énoncés " plus résistants (p. 111). Cette dynamique créative est aussi alimentée par chaque situation, qui « s'accompagne toujours de l'introduction de nouveaux facteurs » (p. 13). On voit que la métaphore poétique apporte un nécessaire correctif au «désir d'efficacité ", qui tend spontanément à confiner chacun dans son poème (Hill, 2009). La poésie est en effet pour K. Burke le lieu même où se cultive la "perspective par incongruité ", donc la possibilité de nouvelles orientations de vie (Anders, 2011): "l'orientation est [trained incapacity], et la ré-orientation est la perspective par incongruité » (p. 69). Se trouver confronté à d'autres façons de voir le monde rouvre et relance la construction de notre poème : « nous pensons la poésie comme l'adoption de stratégies variées pour appréhender les situations ", écrit en ce sens K. Burke (1973, 1).

\section{Conclusion. Images du travail, images du travailleur}

Que peut finalement la métaphore poétique?

D'abord, rendre davantage sensibles, partageables et discutables les traits du travail contemporain. Là où la référence dominante à l'«intensité » entretient l'idée d'un 
travail homogène, sans qualités (Bidet, 2014), elle pointe plutôt sa croissante hétérogénéité interne, qui rend le travail moins aisément repérable (Crague, 2003), ou visible (Crawford, 2010 ; Zweig, 2014) à mesure qu'il est davantage travail d'articulation, d'organisation, d'interface. Derrière la diversité apparente des situations, on peut repérer la généralisation d'une épreuve: "former une orientation " à partir de perspectives hétérogènes, engageant autant de conceptions de ce qui vaut (Dodier, 2015 ; Schwartz, Durrive, 2009 ; Stark, 2011). Et le travailleur de se faire " équilibriste " (Bourgoin, 2015), « funambule » (Gardella, Le Méner, Mondémé, 2006), « explorateur » (Bidet, 2011), « jongleur » (Datchary, Licoppe, 2007), etc.

17 La métaphore poétique offre une voie alternative aux conceptualisations classiques du travail. Là où celles-ci voient des travailleurs à la recherche d'une compensation ou d'un prix de leur travail (salaire, sublimation, statut social), elle voit plutôt des travailleurs à la recherche d'une forme à donner à leur expérience: d'une «ligne d'intérêt active » lui conférant continuité et cumulativité. Cette recherche est d'autant plus vitale que le travailleur est essentiellement mobilisé pour sa plasticité, sa capacité à s'ajuster à des situations constamment singulières, et doit donc organiser constamment son activité. La métaphore poétique aide à lire les contraintes et les ressources du travail en un tel contexte. Mais pour qu'une image aussi forte que celle de Charlot vienne les symboliser, il faudra trouver une autre façon de figurer le corps au travail, qui intègre sa nouvelle immobilité (Rot, Vatin, 2016). K. Burke a souligné le rôle du corps dans la fortune de l'image de Charlot: "l'extrême plaisir procuré par le jeu de Charlie Chaplin venait probablement de ce que son style mimétique précis venait surmonter la confusion sociale. Ses expressions possédaient une signification presque universelle, car elles s'appuyaient sur les certitudes permanentes du corps, les corrélations éternelles entre l'attitude et la posture corporelle » (Burke, 1983, p. 52).

18 En même temps que la production d'une nouvelle image sociale du travail, ce changement de métaphore amène à porter une plus grande attention aux manières dont les travailleurs font face aux contraintes du travail contemporain. Publiciser les efforts qu'ils déploient pour organiser leur activité a aussi pour effet de multiplier les ressources de créativité et d'individuation disponibles. Toute façon de faire, parce qu'elle répond à un environnement ou à des problèmes pour partie similaires, peut en effet être réappropriée et transformée par d'autres : «Les situations sont réelles; les stratégies pour y faire face ont un contenu public; dès lors, les situations se chevauchant d'un individu à l'autre, ou d'une période historique à une autre, les stratégies ont une pertinence universelle» (Burke, 1973, 1). Faire circuler davantage ces stratégies est propre à nourrir la " perspective par incongruité », et à multiplier les « correctifs » aux « trained incapacities ». K. Burke l'illustre ainsi : « Un homme peut tirer du courage d'un poème, en lisant qu'il est le capitaine-de-son-âme ; il peut renforcer cette même assertion, de façon mimétique, en marchant dans la rue aussi vigoureusement que s'il était le capitaine-de-son-âme ${ }^{7}$; ou il peut traduire cette humeur par un ensemble plus complexe de relations en saluant l'une de ses connaissances comme un capitaine-de-son-âme en salue un autre; et tous deux peuvent embarquer sur ce projet comme le feraient deux capitaines-de-leurs âmes » (1983, p. 255).

19 La métaphore poétique ouvre une double piste: développer collectivement une nouvelle image du travail ; et voir les travailleurs comme des producteurs de formes. La première piste peut contrarier la tendance à la fragmentation des mondes du travail ; la 
seconde peut multiplier les ressources d'individuation. Toutes deux incitent à porter le regard là où s'élaborent et s'expérimentent, individuellement et collectivement, ce que K. Burke appelle de "nouvelles propositions de motifs». L'image de Charlot, en dramatisant le travail à partir d'une métaphore mécaniste, laisse au contraire dans l'ombre cette dimension dramaturgique, ou politique du travail - comme exploration en commun de ce qui vaut.

\section{BIBLIOGRAPHIE}

Anders A., 2011, « Pragmatisms by Incongruity: 'Equipment for Living' from Kenneth Burke to Gilles Deleuze », The Journal of the Kenneth Burke Society, vol. 7, $n^{\circ} 2$.

Bidet A., 2007a, « Le travail entre corps et technique : du labor à l'agir créatif », Communications, $\mathrm{n}^{\circ} 81$ - Corps et techniques, Paris, Editions EHESS, 215-223.

Bidet A., 2007b, « Le corps, le rythme et l'esthétique sociale chez André Leroi-Gourhan », Techniques \& culture, $\mathrm{n}^{\circ} 48-49$, p. 15-38.

Bidet A., 2011a, L'engagement dans le travail. Qu'est-ce que le vrai boulot ?, Paris, PUF, Lien social.

Bidet A., 2011b, « La multi-activité ou le travail est-il encore une expérience ? ", Communications, $\mathrm{n}^{\circ}$ 89, EHESS, 9-26.

Bidet A., 2012, « Activité ». Dans A. Bevort, A. Jobert, M. Lallement et A. Mias (coord.). Dictionnaire du travail. Paris, PUF, p. 6-12.

Bidet A., 2014, « Les temporalités de l'activité : rythmicités et styles de vie au travail », in Monchatre Sylvie et Woehl Bernard, 2014, Temps de travail et travail du temps, Publications de la Sorbonne, p. 71-81.

Bidet A., Boutet M., 2013, « Pluralité des engagements et travail sur soi. Le cas de salariés ayant une pratique ludique ou bénévole », Réseaux, La Découverte, vol. 6, n 182, p. 119-152.

Bidet A., Boutet M., Chave F., 2013, « Au-delà de l'intelligibilité mutuelle : l'activité collective comme transaction. Un apport du pragmatisme illustré par trois cas », activités.org, vol. 10, $\mathrm{n}^{\circ} 1$, p. 172-191.

Bidet A., Chave F., 2015, « From Pragmatism to Today's Work Dramas: The Ethicized and Public Dimensions of Work », European Journal of Pragmatism, vol. 7, $n^{\circ} 1$ (en ligne).

Bidet A., Macé M., 2011. « S'individuer, s'émanciper, risquer un style (autour de Simondon) », Revue du MAUSS, 38. Paris, La Découverte : 269-284.

Bidet A., Schoeni D. (éds.), 2011, Dossier « Présences au travail : entre visibilité et invisibilité », ethnographiques.org, $\mathrm{n}^{\circ} 23$.

Bidet A., Vatin F., 2009. « Mesure et acteur au travail », in Steiner Philippe, Vatin François (Eds.) Traité de sociologie économique, Paris, PUF (rééd. augmentée 2013), Quadrige Manuels : 689-718.

Bidet A., Vatin F., 2013. « Le débat sur la souffrance laisse dans l'ombre le travail », Les Cahiers de l'éducation permanente, «Crise du travail, crise dans le travail », $\mathrm{n}^{\circ} 42$, Bruxelles, p. 162-166. 
Bourgoin A., 2015, Les équilibristes. Une ethnographie du conseil en management, Paris, Presses des Mines.

Burke, 1983 (1935), Permanence and change. An Anatomy of Purpose, LA: University of California Press.

Burke, 1973 (1941), Philosophy of the Literary Form: Studies in Symbolic Action, Berkeley: UNiversity of California Press.

Chateauraynaud F., 1997, «Vigilance et transformation. Présence corporelle et responsabilité dans la conduite des dispositifs techniques », Réseaux, 15(85) : 101-127.

Chave F. (2010). Tiers en urgences. Les interactions de secours, de l'appel au 18 à l'accueil en service d'urgences pédiatriques. Contribution à une sociologie du tiers. Thèse de doctorat, sous la direction d'A. Borzeix. Nanterre, Université Paris Ouest-Nanterre La Défense, 13 décembre 2010.

Clot Y., 2010, Le travail à cour. Pour en finir avec les risques psychosociaux, Paris, La Découverte. Crague G., 2003, « Des lieux de travail de plus en plus variables et temporaires », Économie et statistique, 369-370: 191-212.

Crawford M. B., 2010, Eloge du carburateur. Essai sur le sens et la valeur du travail, tr. fr. Paris, La Découverte (2008, Shop Class as Soulcraft: An Inquiry into the Value of Work).

Crawford M. B., 2015, The World Beyond Your Head. On Becoming an Inidvdual in an Age of Distraction, New York: Farrar, Straus and Giroux.

Datchary C., Licoppe C., 2007, «La multi-activité et ses appuis : l'exemple de la 'présence obstinée' des messages dans l'environnement de travail », @ctivités, vol. 4, n 1, p. 4-29.

Dodier N., 1995, Les hommes et les machines. La conscience collective dans les sociétés technicisées, Paris, Métailié.

Gardella E., Le Méner E., Mondémé C., 2006, Les funambules du tact. Une analyse des cadres du travail des équipes mobiles d'aide du Samusocial de Paris, Observatoire du Samusocial, multigr. (repris partiellement in Gardella E., Cefaï D., L'urgence sociale en action. Ethnologie du Samusocial de Paris, Paris, La Découverte, 2011).

Gomez P.-Y., 2013, Le travail invisible. Enquête sur une disparition, Paris, François Bourin.

Crague G., 2003, « Des lieux de travail de plus en plus variables et temporaires », Économie et statistique, 369-370: 191-212.

Hill I., 2009, «'The Human Barnard' and Kenneth Burke's Philosophy of Technologie », The Journal of the Kenneth Burke Society, vol. 5, $\mathrm{n}^{\circ} 2$.

Lorenz E., Valeyre A., 2005, « Les formes d'organisation du travail dans les pays de l'Union européenne », Travail et emploi, $\mathrm{n}^{\circ}$ 102, p. 91-105

Moatty F., Rouard F., Teiger C., 2007, "Lectures pour soi, lectures invisibles ? Une cartographie des lectures au début de l'internet”. Dans A.-F. de Saint Laurent-Kogan et J.-L. Metzger (coord.). Où va le travail à l'ère numérique? Paris, Presses des Mines.

Naville P., 1961, L'automation et le travail humain, Paris, Editions du CNRS.

Rot G., 2014, « Noter pour ajuster. Le travail de la scripte sur un plateau de tournage », Sociologie du travail, vol. $56, \mathrm{n}^{\circ} 1$.

Rot G., Vatin F., 2016, « Surveiller les flux, contrôler les hommes. Du travail et de sa division dansl'industrie chimique et nucléaire ", Sociologie et sociétés (à paraître). 
Schwartz Y., Durrive L., 2009, L'activité en dialogues. Entretiens sur l'activité humaine (2). Toulouse, Octarès.

Sennett R., 2010, Ce que sait la main. La culture de l'artisanat (The Craftsman, 2008). Paris, Albin Michel.

Stark D., 2011, The Sense of Dissonance. Accounts of Worth in Economic Life. NJ: Princeton University Press.

Strauss A., 1988, "The articulation of project work: an organizational process", The Sociological Quaterly. vol. 2, p. 163-178.

Terssac G. (de), 1998, « Le travail d'Organisation comme facteur de performance », Les cahiers du changement, vol. 3 , p. 5-14.

Terssac G. (de), 2006, « Pour une sociologie des activités professionnelles ». Dans Bidet A. (éd.), et al., Sociologie du travail et activité, Toulouse, Octarès, p. 85-100.

Trentin B., 2012, La cité du travail. La gauche et la crise du fordisme, Paris, Fayard.

Tuncer S., 2014, Collaborer et interagir dans les bureaux. L'émergence matérielle, verbale et incarnée de l'organisation, Thèse de doctorat de sociologie, Mines Paris Tech, 11 juin 2014.

Vatin F., 1987, La fluidité industrielle. Essai sur la théorie de la production et le devenir du travail. Paris, Méridiens-Klincksieck.

Vatin F., 1993, Le travail : économie et physique (1780-1830), Paris, PUF, Philosophies.

Vatin F., 1997, “Défense du travail”, réédité dans Travailler est-il (bien) naturel ? Le travail après la fin du travail, Revue du Mauss, $\mathrm{n}^{\circ}$ 18/2, 2001.

Vatin F., 1999, Le travail, sciences et société. Essais d'épistémologie et de sociologie du travail, Bruxelles, Presses de l'Université.

Vatin F., 2007, "Le travail : contrainte productive et contrainte salariale". Dans F. Vatin (coord.). Le salariat. Théorie, histoire, formes. Paris, La Dispute, p. 125-130.

Vatin F., 2008, Le travail et ses valeurs, Paris, Albin Michel.

Vatin F., 2011, “De la définition du travail ou Marx contre Méda”. Dans C. Lavialle (coord.). Le travail et ses régulations. Tours, Presses Universitaires François Rabelais.

Wais E., 2005, “'Trained Incapacity': Thorstein Veblen and Kenneth Burke », The Journal of the Kenneth Burke Society, vol. 2, $\mathrm{n}^{\circ} 1$.

Zweig D., 2014, Invisibles. The Power of Anonymous Work in an Age of Relentless Self-Promotion, New York: Portfolio/Penguin.

\section{NOTES}

1. En 2012-2015, les principales manifestations académiques ou grand public sur le travail ont mobilisé cette iconographie : « Que faire du travail ? ", 4 Printemps des sciences humaines et sociales, Maison européenne des sciences sociales, Lille, 24 mars-12 avril 2012 ; «Quel travail voulonsnous?", Grande Enquête Radio France, Paris, 23 janvier 2012 ; Cycle «Le travail dans tous ses états ", Rendez-vous de crise, EHESS, Paris, 2013 ; Colloque international "L'activité en débat ", Lille, 14-16 janvier 2015. Le même constat peut être fait dans le domaine éditorial.

2. Un nombre déjà conséquent d'auteurs ont entrepris de documenter et de thématiser ces dimensions du travail contemporain. A défaut d'un inventaire (partiel in Bidet 2011a), 
mentionnons quelques travaux français, classiques ou plus récents : Bidet, Boutet, 2013 ; Bourgoin, 2015 ; Chateauraynaud, 1997 ; Chave, 2010 ; Datchary, Licoppe,2004 ; Dodier, 1995 ; Gardella, Le Méner, Mondémé, 2006 ; Naville, 1961 ; Rot, 2014 ; Strauss, 1988 ; Terssac, 1998, 2006 ; Tuncer, 2014 ; Vatin, 1987.

3. "We have tended to consider machinery an absolute good, as illustrated by the frequent identification between mechanization and progress (...) We have been so impressed by the prestige of machinery that we attempt to carry the machine metaphor into other areas of investigation, assuming its absolute or universal interpretative value, as when we employ it to 'explain' kinds of biologic behavior totally different from mechanistic behavior".

4. "The corrective of the scientific rationalization would seem necessarily to be a rationale of art not however, a performer's art, not a specialist's art for some to produce and many to observe, but an art in its widest aspects, an art of living ».

5. «By molding the qualities of our experience, as it sometimes induces us to single out those aspects of events which immediately reflect our interests, and at other times it trains our attention upon the selection of such means as will make events reflect our interests ".

6. La métaphore mécaniste incite elle-même à rabattre ces visions professionnelles sur des « idiotismes de métier ». En l'absence de figures renouvelées et socialisées du travail, les visions professionnelles ont en effet tendance à ne donner de la voix que quand sont menacées, et à privilégier alors la rhétorique nostalgique $\mathrm{du}$ « métier perdu », comme s'il s'agissait de réinvestir ou prolonger des manières de faire issues de temps révolus.

7. En référence probable au dernier vers du court poème Invictus, écrit en 1875 et beaucoup cité, de l'écrivain William Ernest Henley.

\section{RÉSUMÉS}

L'image de Charlot, issue des Temps modernes de Charlie Chaplin, semble incontournable pour illustrer le travail. Cet article vise à comprendre la prégnance de cette image mécaniste, et suggère de s'en défaire pour contribuer à renouveler les façons de figurer le travail. A l'heure où travailler sollicite moins la force motrice des travailleurs que leur capacité à gérer des aléas, les représentations sociales dominantes du travail, issues du laboratoire industriel du XIXe siècle, ne suffisent plus à le mettre en mots, en images, et en partage. Les pistes ouvertes par Kenneth Burke, promoteur d'une "critique culturelle des symboles", suggèrent de substituer à cette métaphore mécaniste une métaphore poétique. Celle-ci permet de décrire les travailleurs comme des producteurs de formes: de manières de travailler et de vivre. En nous rendant attentifs à leurs façons de s'approprier les formes contemporaines de travail, elle nous met aussi sur la voie de nouvelles images du travail.

Charlot's image, taken from Charlie Chaplin's Modern Times, seems unavoidable to illustrate work. This article intends to understand the pervasiveness of this mechanistic representation, and suggests to overcome it in order to help rejuvenate the way work is pictured. While nowadays work require less of workers strength and more of the ability to manage risk, the dominant social representations of work, anchored in the industrial laboratory that was the 19th century, fail to put it in words, pictures and common. Kenneth Burke, as a promoter of a "cultural critique of symbols", suggest a replacement of the mechanistic metaphor by a poetic one. This would offer a description of workers as producers of forms creating new ways to work and live. And by poiting 
out how they take ownership of contemporary types of work, it also puts us on a path towards new images of work.

\section{INDEX}

Mots-clés : symboles, Burke (Kenneth), métaphore mécaniste, métaphore poétique, Chaplin (Charlie), Charlot

Keywords : symbols, Burke (Kenneth), mechanistic metaphor, poetic metaphor, Chaplin (Charlie), charlot

\section{AUTEUR}

\section{ALEXANDRA BIDET}

Chargée de recherche en sociologie au CNRS, Centre Maurice Halbwachs

Alexandra Bidet est chargée de recherche en sociologie au CNRS. Ancienne élève de l'ENS Cachan, agrégée de sciences économiques et sociales, elle développe des recherches qui articulent sociologie du travail, sociologie économique et approche pragmatiste. Elle s'intéresse notamment aux enquêtes que mènent les personnes pour déterminer ce qui vaut, ce qui compte ou ce qui leur importe, aussi bien comme travailleurs, que comme gestionnaires, habitants ou simples passants. Elle est l'auteur de L'engagement dans le travail. Qu'est-ce que le vrai boulot? (PUF, Le lien social, 2011), a coordonné l'ouvrage Sociologie du travail et activité (Octarès, 2006), ainsi que le dossier de la revue ethnographiques.org intitulé "Analyser les présences au travail : visibilités et invisibilités" (2011). Elle a aussi contribué à présenter et traduire en français La formation des valeurs de John Dewey (La Découverte, 2011). 